Sociologie et sociétés

\title{
Idéologie et pratiques syndicales au Québec dans les années '30 : la loi de l'extension juridique de la convention collective de travail
}

\section{Céline SAINT-PIERRE}

Volume 7, numéro 2, novembre 1975

Travaux et recherches sur le Québec

URI : https://id.erudit.org/iderudit/001670ar

DOI : https://doi.org/10.7202/001670ar

Aller au sommaire du numéro

\section{Éditeur(s)}

Les Presses de l'Université de Montréal

\section{ISSN}

0038-030X (imprimé)

1492-1375 (numérique)

Découvrir la revue

Citer cet article

SAINT-PIERRE, C. (1975). Idéologie et pratiques syndicales au Québec dans les années ' 30 : la loi de l'extension juridique de la convention collective de travail. Sociologie et sociétés, 7(2), 5-32. https://doi.org/10.7202/001670ar

\section{Résumé de l'article}

Nous avons choisi de faire l'analyse de la loi de l'extension juridique de la convention collective votée par le Parlement québécois en 1934. Cette loi nous intéresse en tant qu'indice de la pratique des rapports sociaux propre à une société capitaliste : les rapports entre l'État, la fraction industrielle de la classe bourgeoise et la classe ouvrière. Elle nous intéresse aussi par ce qu'elle permet de poser le rapport entre les idéologies et les pratiques syndicales du Québec des années " 30 ". Dans cette étude, nous avons pu rendre compte de cette loi en tant que transcription des idéologies dominantes d'une part mais aussi en tant qu'indice révélateur des orientations normatives et des intérêts politiques de la classe ouvrière, d'autre part. L'étude partielle des débats qui ont entouré cette loi, nous aura permis de mettre en place les forces sociales impliquées dans le développement de la société québécoise de cette époque et de dégager le sens de leur implication.
Tous droits réservés @ C Les Presses de l'Université de Montréal, 1975

Ce document est protégé par la loi sur le droit d'auteur. L'utilisation des services d'Érudit (y compris la reproduction) est assujettie à sa politique d'utilisation que vous pouvez consulter en ligne.

https://apropos.erudit.org/fr/usagers/politique-dutilisation/ 


\section{Idéologie et pratiques syndicales au Québec dans les années ' 30 : la loi de l'extension juridique de la convention collective de travail}

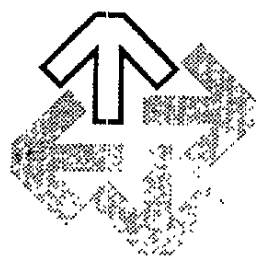

CÉLINE SAINT-PIERRE

"Le fait de l'hégémonie des classes dominantes suppose indubitablement que l'on tienne compte des intérêts et des tendances des groupes sur lesquels l'hégémonie sera exercée, qu'il se forme un certain équilibre de compromis, i.e. que le groupe dirigeant fasse des sacrifices d'ordre économico-corporatif, mais il est également indubitable qu'un tel compromis et que de tels sacrifices ne peuvent concerner l'essentiel... 1 "

\section{NOTE PRÉLIMINAIRE}

Ce texte constitue un extrait d'un travail de recherche portant sur «l'analyse des orientations et des pratiques du syndicalisme catholique et des unions internationales, la définition des idéologies dominantes et la mise à jour des contradictions fondamentales » qui ont marqué la société québécoise des années «1930$1940 » .11$ va sans dire que plusieurs des éléments nécessaires à la compréhension et surtout à l'explication de notre objet d'étude sont absents de ce texte où nous tentons d'analyser un des niveaux de la pratique des rapports de classe propres

1. Gramsci, A., Note sul Machiavelli sulla politica e sullo stato moderno, Editions Enaudi (Citation recueillie dans Poulantzas, N. Pouroir politique et classes sociales Maspero, 1968). 
à une société capitaliste, celui des rapports patrons-ouvriers dans l'entreprise industrielle. C'est une loi, celle de «l'extension juridique de la convention collective», adoptée en 1934 qui joue ici le rôle d'indice privilégié dans la conduite de notre analyse.

\section{INTRODUCTION}

Une étude préalable (dont cet article ne présente qu'une partie) nous a permis de mettre à jour lc cheminement suivi par le projet de l'extension juridique de la convention collective de travail, projet qui s'est transformé en loi, c'est-à-dire en une pratique d'organisation des travailleurs et de réglementation juridique des rapports sociaux propres à l'entreprise industrielle et commerciale de type capitaliste. Nous allons nous intéresser ici plus particulièrement à en saisir le sens et les objectifs d'une part, et d'autre part à analyser le degré de concordance qui existe entre ces objectifs et l'application réclle de la loi, application qui prend la forme des décrets.

Nous considérons la loi (dans son sens général) comme une transposition à un certain niveau (sens spécifique) de la forme d'organisation sociale prévalant dans une société donnée, elle-même informée par un mode de production dominant. Cette forme d'organisation sociale est sous-tendue par un affrontement permanent entre des agents dont les intérêts sont en contradiction les uns avec les autres et la loi peut être considéréc comme un mécanisme de gestion des compromis entre intérêts antagonistes, compromis plus ou moins acceptés par les parties en cause, suivant que le rapport de force leur est favorable ou pas. Une loi n'opère donc pas une réduction des intérêts en jeu mais elle permet un déplacement du lieu d'affrontement d'intérêts contradictoires. Elle définit le terrain sur lequel les agents peuvent se rencontrer et s'affronter et fixe le seuil de leur affrontement. La délimitation de ce seuil constitue une barrière qui ne peut être franchie par l'une ou l'autre partie sans entraîncr des transformations radicales du système social établi. Cette définition que nous donnons de la loi trouve son application, il va sans dire, dans les sociétés capitalistes dites de «démocratie libérale ». Il nous faudrait pour être juste, revoir cette définition en regard des pays fascistes et des pays socialistes.

Étant donné le type de société qui nous concerne (capitaliste), la loi peut être analysée en tant que transcription des idéologies dominantes c'est-à-dire des idéologies des classes sociales qui détiennent le pouvoir politique et économique dans cette société ; mais elle est, en même temps révélatrice des orientations normatives et des intérêts politiques de la classe dominée. Pour la période qui nous intéresse (1929-1940) les fractions de la classe dominée qui seront touchées par la loi de 1934 sont constituées principalement par les ouvriers de métier et d'industrie et par les employés du secteur privé des services. Pour ce qui est des classes dominantes, elles sont composées d'une part de la bourgeoisie industrielle, financière et commerciale et de la petite-bourgeoisie québécoises et d'autre part, de la grande bourgeoisie surtout financière (elle réunit les représentants du capital bancaire et $\mathrm{du}$ capital industriel) majoritairement américaine et anglo-saxonne et en laquelle se 
fond une grande partie de la bourgeoisie québécoise $\%$ Nous considérerons la loi de l'extension juridique de la convention collective et les débats qui l'ont cntourée et préparée en tant qu'indices privilégiés nous permettant d'une part, de mettre en place les forces sociales impliquées dans le développement de la société québécoise de cette époque et de dégager d'autre part, le sens de leur implication.

Jacques Perreault ${ }^{3}$ mentionne trois aspects de l'évolution juridique au Québec :

1. Les ouvriers canadiens-français ainsi coupés de tout lien avec leur milieu culturel sont moins conscients que les ouvricrs américains d'un besoin d'institutions et de règles juridiques nouvelles pour supprimer les maux dont ils souffrent.

2. L'ouvrier canadien-français a été éveillé par les ouvriers étrangers. C'est le syndicalisme américain qui est venu dire aux ouvriers canadiens-français : "Organisez-vous en syndicats », "établissez chez-vous au moins deux institutions : le syndicalisme et la convention collective $\gg$. On les a établies de 1858 à 1930, à la façon de tous les pays industricls, i.e. avec assez de violence.

3. Cette attitude de la classe ouvrière dirigée par des chefs syndicalistes américains ou des chefs canadiens formés au syndicalisme américain, a provoqué une vive opposition, et une forte animosité de la part de la classe rurale et des classes moyennes canadiennes-françaises. En conséquence, le pouvoir politique dominé par les classes rurales et par les classes moyennes n'a légiféré dans le domaine industricl qu'à contre-cœur.

Jusqu'en 1898, les tribunaux québécois ne voulurent pas admettre l'action du syndicalisme et il fallut une décision de la Cour Suprême du Canada (dans la cause de Perreault vs. Gauthier, 28 can. S.C.R. p. 241) en 1898, pour que les tribunaux de la Province de Québec admettent l'existence des syndicats ouvriers. Il faudra même attendre une décision de notre cour d'appel, en 1942, pour que les tribunaux de la Province de Québec affirment une seconde fois at de façon non équivoque, la parfaite légalité du syndicalisme ouvrier ${ }^{4}$.

Cette analyse de Jacques Perreault concernant l'évolution juridique et la situation socio-économique des Canadiens-français nous semble intéressante à plusieurs points de vue et éclaire d'une certaine manière les problèmes que nous étudions. D'abord elle appuie notre hypothèse voulant que la législation dans une société constitue un indice privilégié pour saisir les oricntations des agents en présence : les orientations se situant au pôle : mouvement social et les lois au pôle : institutionnalisation, organisation sociale, d'après la dichotomie établic par Alain Tourraine dans l'élaboration de la «Sociologie de l'Action ». Lcs premières ne se fondant pas nécessairement dans les secondes à cause des contradictions qu'elles véhiculent, c'est pourquoi il nous faut donc dépasser l'analyse de l'institution pour déceler les contradictions qu'elle ne révèle pas cn première analyse. Tout comme les idéologies, la législation joue souvent le rôle de masque et n'exprime pas les véritables débats qu'animent les agents qui s'opposent les uns aux autres. Ceci ne détruit pas pour autant ce que nous avons dit précédemment concernant l'intérêt de l'analyse cle la législation d'une société mais exige cependant que nous la dépassions.

2. Aucune recherche concrète n'ayant été menée de façon rigoureuse sur la classe bourgeoise au Canada et au Québec, notamment pour cette période, nous admettons le caractère hypothétique de notre assertion développée dans un autre chapitre de notre recherche.

3. Perreault, Jacques. L'évolution juridique in Essais sur te Quélec contemporain, P.U.L., 1953.

4. Perreault, J., op. cit., p. 130 et suivantes. 
C'est pourquoi nous devons voir la/les lois(s) comme un effet d'une mise en rapport des idéologies dominantes et des orientations normatives de la classe ouvrière mouvement ouvrier organisé en l'occurrence) lesquelles induisent des pratiques de classe et inversement. L'explication que donne J. Perreault du retard de la législation du travail sur la situation objective dans laquelle se trouve le Québec des années 1900-1930 doit être située dans cette problématique.

Cependant, le sens de la loi de 1934 ne se saisit pas uniquement en faisant appel à l'analyse des idéologies dominantes et des pratiques de classe saisies à travers le rapport ici privilégié : patronat capitaliste et classe ouvrière dans sa fraction organisée en syndicats. Il nous faut aussi connaitre les composantes de l'infrastructure économique ct ses particularités à ce stade donné du développement du Québec car c'est sur cette base que se spécifient les rapports de classe.

La loi de 1934 définit justement 1. le type de rapports patrons-ouvriers soit la négociation en vue d'aboutir à une convention collective; 2 . les agents: patrons et ouvriers signataires d'une convention collective et les tiers auxquels s'étend cette convention et qui ne sont pas nécessairement syndiqués ni signataires de la convention initiale, n'ayant pas participé à la négociation ; 3. l'enjeu à propos duquel les acteurs s'affrontent: salaires, heures de travail, apprentissage; 4 . le caractère obligatoire au niveau de l'application de la convention collective signée. Le comité paritaire se voulant l'expression du principe suivant lequel patrons et ouvriers gèrent eux-mêmes leurs rapports; 5 . le rôle de l'État qui apparaît prépondérant et qui, d'une certaine façon peut détruire, annuler ou neutraliser le rôle du comité paritaire et les revendications des travailleurs et des cmployeurs.

L'orientation que prend la définition des rapports entre patrons et ouvriers à travers le régime de la convention collective doit donc être analysée en fonction du contexte économique et politique de la société concernée. Car le régime de la convention collective extensionnée est le reflet de la société qui le produit et il a à son tour des répercussions sur les orientations économiques, politiques et sociales de cette même société. Cependant nous verrons dans l'analyse subséquente que nous ferons du processus qui a conduit à la loi de 1934 que les patrons et les ouvriers sont des agents partiels de ce mouvement sous-jacent aux principes de la loi. Si nous nous étions bornés à étudier le contenu de la loi nous n'aurions jamais pu en saisir le sens et la signification, nous n'aurions jamais pu en découvrir les véritables agents. Cette démarche que nous utilisons tend à démontrer, par l'analyse d'un cas particulier, les limites d'une étude des rapports patrons-ouvriers en termes de « relations industrielles $»$. À ce niveau de l'analyse, la «convention collective » ou tout autre mode de règlements des conflits de travail (dont les lois sont des transcriptions) sont conçus comme des instruments ou des méthodes qui règlent les relations entre les patrons et les ouvriers. Pour aller au-delà de cette analyse, il nous faut prendre une autre méthode, partir sur un autre picd. II nous faut commencer par l'analyse des rapports fondamentaux qui régissent la société dans son ensemble pour aboutir à l'étude de ses éléments constituants : en l'occurrence, le mouvement ouvrier et ses rapports avec les autres éléments constituants de cettc société.

La loi de 1934 est donc une réponse à une situation donnée (à une conjoncture déterminée) : la crise économique de 1929 et le développement de la grande industric capitaliste dans la forme qu'il prend au Québec, soit l'impérialisme, mais 
elle est en même temps la manifestation d'une volonté de participation au développement économique et de contrôle de ces objectifs. En tant que loi, elle se situe dans le cadre de l'institution (pôle institutionnel) et en tant qu'expression d'une prise en charge du développement de la société par une fraction de la classe ouvrière et de la petite bourgcoisie, elle se rapproche du pôle du mouvement social.

Cette loi de 1934 et le principe d'organisation des rapports patrons-ouvriers qu'elle instaure est née à la fois d'une volonté du syndicalisme ouvrier catholique de faire reconnaître ses droits mais aussi de la rencontre de cette volonté avec une infrastructure économique qui en période de crise provoque un déséquilibre au niveau des forces sociales qui doivent s'orienter différemment pour répondre à leur objectif de contrôle du développement.

Dlans les années qui suivirent la mise en vigueur de la loi des syndicats professionnels, les syndicats catholiques, surtout ceux incorporés en vertu de la loi de 1924 , tentèrent de conclure des conventions collectives de travail avec lis employeurs. Ils réussirent dans un certain nombre de cas, mais dans la majorité, ils se butèrent à des échecs. Surtout dans les secteurs industriels où le métier n'était pas fortement organisé. Ce fait venait pour une bonne part, du refus obstiné des patrons à se départir de leurs pouvoirs discrétionnaires et de l'attitude plutôt hostile qu'ils adoptèrent vis-à-vis de la loi de 1924. Mais la raison majeure pour laquelle les syndicats ne réussirent pas à négocier collectivement dans la mesure où ils l'auraient voulu résidait avant tout dans la faiblesse interne de la loi, laquelle ne prévoyait que des conventions collectives particulières, c'est-à-dire n'cngageant que les parties contractantes. Si l'on ajoute à cela qu'une tclle convention, en plus d'être et de rester particulière, reposait sur une base absolument facultative, l'on conçoit vite le champ restreint où elles opéraicnt.

$\grave{A}_{\mathrm{L}}$ cette situation vinrent s'ajouter, à partir des années 1929 et 1930, les complications apportées par la dépression économique et la baisse générale des affaires. L'industrie est dans le marasme, le chômage grandissait, les prix s'affaissaient. Les industriels ne veulent point signer de contrat collectif de travail qui les obligerait, par les voies de la négociation, à consentir des salaires plus élevés que ceux payés par ses concurrents. Les employeurs liés par les coinventions collectives, se trouvaient vis-à-vis de leurs concurrents en position d'infériorité 5 .

L.a crise n'atteint pas seulement les individus mais les associations de toute sorte, en particulier les syndicats. Gérard Tremblay, devenu sous-ministre du travail, est remplacé par son beau-frère, Léonce Girard, au secrétariat des syndicats catholiques. Tous deux propagent l'idée de l'extension juridique des conventions collectives. Mais des syndicats s'étiolent.

Des chômeurs ne versent pas leur cotisation, d'autres se sentent trop à la merci des patrons pour lutter... Le découragement se répand dans les « fraternités 》 de la chaussure, si éprouvées à Québec. L'abbé Maxime Fortin veut sauver ces syndicats par le système des contrats collectifs et des ateliers fermés. Mgr Villeneuve (autorité religieuse de la province de Québec) recommande ct même commande aux communautés et institutions religieuses d'accorder la préférence aux patrons ayant conclu un contrat collectif avec des syndicats catholiques".

5. Cardin, Jean-Réal, L'influence du syndicalisme national catholique sur le droit syndical québécois in les Cahiers de l'Institut Social Populaire - juin 1952, p. 30.

6. Rumilly, R., Histoire de la Province de Québec, Ed. Fides, Montréal, p. 110. 
Ce qui importe de retenir c'est que le mouvement qui a conduit à penser un nouveau système d'organisation des rapports patrons-ouvriers s'est amorçé avant la période de la crise économique.

Ce qui apparaît cependant avec assez de clarté c'est que la situation économique apparaît déterminante dans l'explication du fait de l'adoption de cette loi à ce moment précis de l'évolution de la société québécoise. Il est probable qu'en temps de prospérité, cette loi n'aurait probablement pas été votée car le syndicalisme, et c'est là l'opinion des Unions Internationales, de par sa force économique, aurait pu parvenir à «maintenir un certain équilibre ». En temps de crise, cela devient difficile et impossible, car les contrats de travail que les syndicats passent avec leurs employeurs, ne couvrent pas un champ d'action assez vaste pour influer sur le marché du travail.

Nous nous proposons maintenant de faire une étude plus approfondie des positions adoptées par les organisations syndicales dans ce débat particulier autour de la loi de l'extension juridique de la convention collective. Rappelons que l'analyse de cette législation poursuit un double objectif : repérer la loi comme indice de la pratique des rapports sociaux dans l'industrie et le commerce d'une part et d'autre part, en autant que son application le permet, rendre compte du degré de cohérence qui existe entre l'idéologie corporatiste et la pratique syndicale de la C.T.C.C. et entre l'idéologie de la démocratie industrielle et la pratique syndicale du C.M.T.C. au Québec. De plus, la loi de 1934 ayant provoqué un affrontement au niveau de la pratique syndicale entre la C.T.C.C. et le C.M.T.C., il s'agit donc de rendre compte du contenu de cet affrontement et de montrer jusqu'à quel point il révèle une référence aux idéologies propres à chacune des organisations.

\section{LA CONFÉDÉRATION DES TRAVAILLEURS CATHOLIQUES DU CANADA (C.T.C.C.) ET L'EXTENSION JURIDIQUE DE LA CONVENTION COLLECTIVE}

Dans notre étude des origines de la loi de 1934, nous avons pu constater le rôle prépondérant du syndicalisme catholique dans la définition de son principe et de sa mise en application. Il ressort donc que la C.T.C.C. prône cette loi et en exige l'adoption par le gouvernement provincial pour en légaliser le recours tant de la part des syndicats que du patronat.

Sur le plan de la législation ouvrière, la C.T.C.C. présente cette loi comme novatrice en Amérique du Nord. Les autres provinces anglaises ne connaissent pas pareil système et les États-Unis pour leur part viennent de voter le N.I.R.A., qui sera déclaré inconstitutionnel en 1935.

Considérées sous un autre angle, les orientations du syndicalisme catholique sont guidées par l'idéologie corporatiste introduite par le clergé catholique qui essaie de promouvoir la bonne entente entre employeurs et salariés. Cette forme de syndicalisme orienté vers le corporatisme constitue une base d'intégration t d'adaptation à la société industrielle de type capitaliste tout en marquant une forme d'opposition au développement du capitalisme sur une grande échelle contrôlé par des intérêts étrangers, «extérieurs à la société québécoise ». 


\subsection{LES IDÉOLOGUES DE LA C.T.C.C.}

C'est dans la doctrine sociale de l'Église Catholique que se retrouvent les principes de base du corporatisme ct le clergé s'en fait le principal interprète. Voyons comment la loi de '34 est l'application ou plutôt la pratique de cette idéologie. Les Jésuites et certains intellectuels ont participé de près à la diffusion de cette idéologie. Voyons comment ils considèrent la loi de '34 dans son rapport avec cette idéologie, fondement des orientations du syndicalisme catholique :

La loi de 1934, constitue l'une des lois qui ont été les plus efficaces dans notre province ; elle a permis et favorisé le rapprochement entre patrons et ouvriers organisés, cartel forcé au début où il y avait plus d'antagonisme que d'amitié. La loi a amené de fait des associations autour des tables, surtout dans les comités paritaires, certes, il s'est trouvé des patrons pour s'intéresser outre mesure au syndicalisme de boutique, unions de compagnie. Patrons et ouvriers cnt pris conscience de leur communauté. Pie XII insiste sur la communauté entre patrons et ouvriers qui se fait autour du travail ; les comités paritaires et la pratique de la convention collective à extension juridique ont au moins donné cette impression qu'on y protège ensemblc un patrimoine commun... Ainsi dans la conception $d u$ syndicalisme ou des relations du syndicalisme avec l'Etat en particulier s'observent les plus radicales transformations. Le syndicalisme d'autrefois, organisation presque individuelle a fait place à un syndicalisme intégré dans l'entreprise et dans l'État ${ }^{\top}$.

2.1.1. Le Père Archambault qui est à l'origine du mouvement de l'École Sociale Populaire, définit ainsi le sens de la loi de $1934^{\circ}$ :

Bienfaisante pour l'ouvrier, cette mesure, comme la plupart de celles qu'ont préconisées les syndicats catholiques, l'cst aussi pour les patrons. Elle les cébarrasse d'une concurrence ruineuse, elle leur assure une main-d'œuvre compétente et bien disposéc, elle écarte les conflits de leurs usines. Et elle contribue ainsi au maintien de l'ordre social. L'autre aspect de cette loi c'est cu'elle oriente notre régime social vers l'ordre corporatif.

Modelé sur le type que lui offrait l'illustre moraliste belge, le R.P. Vermeersch, le syndicalisme catholique au Canada, après avoir relevé la situation de l'ouvrier et barré la route à la révolution, s'apprête à compléter son cycle bienfaisant en préparant l'avènement de l'ordre corporatif, d'après les directives de l'Encyclique Quadragesimo Anno.

Quant au Père Vermcersch, cité par Archambault comme ayant influencé le syndicalisme catholique au Canada, il définit ainsi le syndicalisme :

Le vrai syndicalisme est d'abord:

1. un organe de défense professionnelle. Le patron, c'est un groupement impersonnel, sans âme et sans conscicnce pour qui seuls les profits comptent. Tout est subordonné au rendement pécuniaire de l'entreprise, même le bienêtre temporel, même la vic morale de l'employé. Pour la foule des travailleurs, il n'est pas de rempart meilleur que le syndicat.

2. un instrument de pacification sociale. Défense professionnelle ne signific pas nécessairement lutte des classcs. Lorsque surtout les intérêts se trouvent liés en quelque sorte les uns aux autres comme le sont dans une industrie ceux de l'employcur et de l'employé, leur respect mutuel ne peut-être qu'utile.

7. Cousineau, Jacques, s.j., L'Evolution de la mentalité sociale au Québec depuis 1931, Institut: Social Populaire, $\mathrm{n}^{\circ} 487,1956, \mathrm{p} .16$.

8. Archambault, J.P.. Te Syndicalisme catholique an Canada. Ecole Sociale Populaire, $\mathrm{n}^{\circ} 26 \%, 1936$, p. 21-23. 
3. il doit être chrétien : la foi attache le syndicat à l'Église catholique.

4. il doit être national, d'abord centré sur les problèmes du milieu (se référer à la constitution de la C.T.C.C.) ${ }^{9}$.

2.1.2. Esdras Minville, à qui nous avons consacré une partie de notre analyse sur le corporatisme et en qui il faut voir un collaborateur de l'École Sociale Populaire, envisage ainsi le sens de la convention collective :

Des diverses dispositions de la législation ouvrière, la convention collective (y compris la convention à extension juridique) est probablement la plus complètement en contradiction avec l'esprit qui a animé le monde des affaires et le monde ouvrier jusqu'en ces tous derniers temps. Il importe d'interpréter à sa juste valeur le mouvement qui, à l'heure actuelle, en fait réclamer partout la généralisation; car la convention collective peut être un régime ou un simple mécanisme. Elle sera un simple mécanisme si au lieu d'être pratiquée dans l'esprit dont elle-même procède, elle est pratiquée dans l'esprit traditionnel et du syndicalisme ouvrier - esprit de lutte, d'antagonisme - et du monde des affaires - esprit de domination et d'assujettissement. Si la convention collective doit devenir en quelque sorte l'institution centrale d'un juste régime ouvrier, il faudra de part et d'autre la pratiquer dans un esprit de collaboration. L'employeur devra donc de son côté considérer l'ouvrier, non comme un accessoire, une pièce d'outillage dont il s'agit d'obtenir le plus haut rendement, mais comme un collaborateur qui a droit à une juste rémunération de ses services, à la permanence de son emploi et à des conditions de travail qui ne le menacent ni dans sa santé physique ni dans sa santé morale. Il lui faudra retrouver un peu de l'esprit des anciennes corporations, alors que l'artisan faisait en quelque sorte partie de la famille même de son maître et était traité comme tel. Il se rendra vite compte que la politique la plus sociale est en définitive la plus sage. Cette adaptation d'esprit, il faut aussi l'attendre des employés. La convention collective ne doit pas être considérée par eux comme un moyen additionnel de prendre barre sur le patron, mais comme un moyen de régulariser leurs relations avec lui au grand jour de la loi. La première preuve à attendre des ouvriers qu'ils comprennent véritablement l'esprit de la convention collective, c'est la constitution légale de leur syndicat et ainsi l'acceptation ouverte et pleine de responsabilités découlant et pour les individus et pour les groupes, de la signature d'un contrat ${ }^{10}$.

2.1.3. Un autre économiste, Adrien Gratton ${ }^{11}$, parle de l'extension juridique des contrats collectifs de travail comme "d'un pas très important vers le corporatisme social». Les dispositions prévues par la loi supposent le «principe de la collaboration entre les différents producteurs exerçant une même fonction économique, puis entre ceux-ci et leurs employés $\gg$.

Il ressort done de ces textes que nous venons de présenter ct qui s'avèrent représentatifs de l'orientation de l'E.S.P. concernant le principe de la loi de 1934, que le corporatisme social demeure le fondement qui règle l'organisation des rapports patrons-ouvriers. Voyons maintenant comment le syndicalisme catholique se définit par rapport à cette idéologie. D’une façon générale, le syndicalisme

9. Vermeersch, A., Ce que nous entendons par Syndicat, syndicat chrétien, extraits reproduits dans Archambault, J.-P., op. cit., p. 3.

10. Minville, Esdras, Pour une doctrine ouvrière, in Actualités Economiques, janvier 1944 , p. 272-274.

11. Gratton, Adrien, L'orientation de la Province de Québec vers le corporatisme social in Actualités Economiques, août-septembre 1937, p. 341. 
catholique de son côté adhère entièrement au Programme de Restauration Sociale construit par le mouvement de l'École Sociale Populaire:

Depuis le dernier congrès (1932), nos ouvriers ont été sollicités par une foule d'organisations enseignant les doctrines les plus diverses. Nous avons des unions neutres, nous avons des fédérations, des clubs, nous avons des socialistes, des communistes, des technocrates et que sais-je encore. Il faut que ce congrès (1933) définisse bien sa doctrine. Il faut qu'il travaille à l'expansion de ses cercles d'études et qu'il voit à l'organisation d'une propagande active et constante.

Nous devons donner notre entière adhésion au Programme de Restauration Sociale (de l'École Sociale Populaire) rédigé dernièrement et faire connaître au grand jour notre doctrine de justice, de charité, de collaboration des classes. Nous devons travailler à ce que soit assuré aux ouvriers le bien-être. Four cela, demandons l'aide de l'État, mais travaillons surtout à ce que les professions soient bien établies et à ce que les ouvriers de notre pays jouissent de la protection des contrats de travail ${ }^{12}$.

2.1.4. Alfred Charpentier l'un des premicrs présidents de la C.T.C.C. et l'un de ceux qui a contribué le plus à définir ses oricntations et sa pratique, définit ainsi le rôle du syndicalisme:

Le but essentiel du syndicat est donc de négocicr le contrat collectif de travail. Cela dans l'entreprise individuclle. Au-dessus de cette entreprise, il doit viser à obtenir une convention collective avec toutes les entreprises d'une même profession et ainsi tendre à l'organisation complète de la profession. Sur ces cleux plans, c'est exclusivement des intérêts économiques, sociaux et moraux de ses membres qu'il doit s'occuper. Il doit respecter les frontières du syndicalisme et de la politique, laisser ses membres prendre position individuellement sur le plan politique...

Certains chefs syndicalistes s'opposent à l'orientation des syndicats vers l'organisation professionnelle corporative, craignant qu'une évolution en ce sens entraîne la disparition des syndicats. C'est une profonde erreur car, disait Pie XII, "l'institution corporative donnera un couronnement naturel aux organisations syndicales $\gg$.

Ce n'est que par l'organisation corporative que les travailleurs, par leurs représentants syndicaux, «assumeront leur part de responsabilité dans la bonne ordonnance de la profession et dans la constitution et le développement de l'économie nationale ». (Pie XII). Il faut comprendre que le syndicat ouvrier n'est qu'un instrument pour organiser une partie de la profession, la partie ouvrière et que la seconde partie doit l'être par le syndicat patronal; il faut comprendre aussi que la profession est au-dessus des deux syndicats et qu'un organisme paritaire permanent doit la personnifier pour y promouvoir des réalisations de bien commun. L'institution corporative n'est pas autre chose. C'est vers ce but que doit tendre le syndicalisme pour répondre à l'enseignement social de l'Église ${ }^{13}$.

Le régime économique avait été dépouillé de l'esprit chrétien selon Charpentier, il ne sera donc possible de revenir à un «régime vraiment humain dans le monde industriel que dans un régime de juste milieu qui n'étouffe pas la liberté humaine mais qui lui fixe des limites raisonnables».

12. Filion, Osias, président de la C.T.C.C. - in Procès-Verbal du Congrès de la C.T.C.C., Montréal 1933.

13. Charpentier, Alfred, Un enseignement méconnu in la revue Relations, septembre 1959, p. 233. 
Ce système humain, seule la collaboration entre l'Église, l'État et la profession organisée pourra le donner :

L'Église a comme mission de faire descendre la paix sur terre et de faire respecter les règles immuables de la morale chrétienne.

L'État a comme rôle de rechercher le bien commun des individus et de la nation et l'harmonisation des rapports économiques et sociaux entre les classes.

L'organisation professionnelle qui n'est pas simplement l'association ouvrière ou patronale, mais bien la réunion des deux dans la chambre paritaire locale, provinciale ou nationale. C'est là la corporation professionnelle.

Cet aménagement social entre le pouvoir politique et les corps professionnels ne sera rendu possible que si l'État et les corps professionnels sont chrétiens. La loi de l'extension marque déjà le commencement du système corporatif ${ }^{14}$.

Ce qu'il importe de relever de cette définition du syndicalisme catholique par un de ses leaders les plus importants c'est la reconnaissance du corporatisme comme seule base possible des relations patronales-ouvrières d'une part, et d'autre part la délimitation du rôle du syndicalisme qui fait de ce dernier une institution centrée sur la défense des «intérêts économiques, sociaux et moraux» des travailleurs et dont la convention collective demeure le mode d'organisation de cette défense d'intérêts. Quant aux orientations politiques, elles ne sont pas du ressort du syndicalisme mais demeure au niveau de la liberté individuelle.

\subsection{LE JOURNAL : LA VIE SYNDICALE}

Nous allons maintenant étudier la position du syndicalisme catholique à travers son journal, La Vie Syndicale, afin de voir s'il y a continuité ou rupture entre les producteurs de l'idéologie corporatiste et ceux qui la transmettent aux travailleurs.

Pour les syndicalistes catholiques, la loi de l'extension juridique de la convention collective demeure l'une des mesures les plus importantes à l'intérieur $d u$ Programme de Restauration Sociale.

L'extension juridique est supérieure au projet américain car elle donne aux organisations patronales et ouvrières leur véritable rôle. Notre projet (celui du Syndicat catholique) semble atteindre parfaitement l'idéal tracé par la doctrine sociale de l'Église en ce scns que toutes les conditions de travail sont déterminées par ententes entre patrons et ouvriers. Le gouvernement contrairement à ce qui se fait aux États-Unis, n'élaborera pas les codes ; il travaillera seulement à ce que des ententes s'effectuent et donnera aux contrats de travail une valeur générale sur toute l'industrie intéressée. De cette façon, l'État assurera la collaboration étroite et incitera employés et employeurs à s'entendre, à traiter ensemble et à coopérer.

La passation de cette mesure sera pour les syndicals qui s'inspirent de la doctrine catholique, un véritable triomphe après 15 ans de rudes combats.

Ce sera un grand pas vers une véritable restauration de l'ordre social et vers l'institution de la corporation, qui est à l'opposé mêrme du socialisme ${ }^{1 \bar{v}}$.

14. Charpentier, Alfred, La Vie Syndicale, vol. XIX, ${ }^{\circ} 17$, juillet 1935, p. 7-8.

15. Girard, Léonce, in La Vie Syndicale, vol. XIII, $\mathrm{n}^{\circ} 11$, janvier 1934. 
L'organisation des rapports patrons-ouvriers par le recours à la législation est définie comme «anti-naturelle », comme allant contre l'orientation définie en termes de « développement harmonieux $»$ des rapports patrons-ouvriers, orientation qui privilégie le rôle du patron en l'homologuant à celui de «père» dans la famille. Cette législation est donc définie comme un palliatif permettant de corriger une situation où les patrons ne s'acquittent pas de leur rôle tel que défini au sein de celte idéologie. Ce qu'il faut faire remarquer ici et qui nous permette de développer un point de l'analyse sur l'idéologie corporatiste, c'est la référence au 《naturel », à la «nature »: «méthodes naturelles», «droit naturel ». Ce qui est présenté comme tel par la doctrine sociale de l'Église Catholique et qui se transpose au niveau de l'idéologie corporatiste, correspond en fait à une vision du monde très spécifique de certaines catégories sociales qui portent leur action au niveau des mécanismes qui seraient susceptibles selon eux d'améliorer les rapports sociaux (législation, arbitrage, conciliation) et non au niveau de la remise en cause de la structure de la société fondée sur les classes sociales, sur les rapports sociaux existants dans une société industrielle de type capitaliste. Le « naturel», le "conforme à la nature » doit alors être englobé dans l'idéologique, en tant que support de cette idéologie.

\subsubsection{L'extension juridique de la convention collective comme principe d'orga- nisation des travailleurs}

«L'extension juridique... tend à solutionner le problème angoissant de notre époque, celui de l'incertitude de la vie du prolétaire ».

Pour le syndicalisme catholique, la solution de ce problème ne se trouve pas «dans le libéralisme économique » ou dans «la destruction du capital», mais dans «la collaboration étroite de tous les groupes de la société, dans une restauration de l'ordre social conforme à la doctrine chrétienne ${ }^{16}$ 》 et c'est pour atteindre ce but que le syndicalisme a réclamé du gouvernement l'extension juridique de la convention collective. Par cette loi, on octroie à l'État un rôle central.

L'État, dans une société bien organisée, doit diriger, surveiller, stimuler et contenir. À l'organisation intermédiaire (les syndicats patronaux et ouvriers en sont) appartient de régler toutes les questions de moindre importance ${ }^{17}$.

L'extension juridique de la convention collective apparaît, pour les syndicalistes catholiques, comme directement responsable de l'augmentation des salaires de 7 à 8 millions de dollars pour environ 150,000 ouvriers et ce en l'espace de quatre ans (1934-1938). Cette loi est la première victoire, insigne sans précédent en notre province, contre l'individualisme et le libéralisme économique. Son adoption supposait d'abord "l'édification d'un mouvement syndical ayant le prestige d'avoir toujours diffusé dans le peuple les principes et les idées les plus sains, les plus justes, les plus clairvoyants et les plus constructifs du bon ordre social ${ }^{18} \gg$.

A.u principe de l'extension juridique s'ajoute la pratique de l'arbitrage obligatoire comme moyen de réglementation des conflits entre patrons et travailleurs.

16. Girard, Léonce, in La Vie Syndicale, vol. XIV, $\mathrm{n}^{\circ} 2$, avril 1934, p. 8.

17. Girard, Léonce, op. cit., p. 8.

18. Charpentier, A., in La Vie Syndicale, vol. XXII, $n^{\circ} 9$, mai 1938, p. 1. 
Ceci afin de permettre aux travailleurs de faire cntendre leurs réclamations et d'obliger les patrons à les discuter. Il serait ainsi possible selon les proposeurs de cette mesure d'éviter des situations comme celles :

a) des imprimeurs de Montréal qui depuis deux ans travaillent à généraliscr un contrat de travail à leur région ;

b) des travailleurs de Thetford Mines et d'Asbestos, dont le patron a tenu en échec la demande des ouvriers dont $90 \%$ étaient groupés en syndicat;

c) des travailleurs de la Dominion Textiles à Valleyficld, Louiseville.

Ce que nous voulons par l'arbitrage obligatoire c'est simplement de supprimer les dangers de grève et de conflit, pour les remplacer par les décisions de bureau d'arbitrage. Nous voulons un autre moyen que la grève, plus conforme au bon ordre social et plus acceptable dans un pays civilisé, soit la conciliation et l'arbitrage ${ }^{13}$.

L'élimination du conflit ou le règlement de celui-ci par des moyens qui favorisent la coopération et la bonne entente doivent être considérés comme des orientations de l'action du syndicalisme catholique lorsque celui-ci propose des principes d'organisation des travailleurs comme l'extension juridique de la convention collective, la conciliation et l'arbitrage obligatoire des conflits patronstravailleurs.

Le syndicalisme catholique voit dans ce principe d'organisation des travailleurs, des avantages à la fois pour ceux-ci et pour les employcurs :

a) Avantages pour l'employeur

1) l'extension juridique met fin à la concurrence malhonnête, faite surtout sur le salaire;

2) elle lui donne confiance en sa main-d'œuvre ;

3) elle lui assure une main-d'œuvre satisfaite qui lui donnera $100 \%$ de rendement qui évitcra le gaspillage de matériel ;

4) elle lui permet de s'unir pour faire disparaître les contracteurs irresponsables ;

5) elle enlève toute responsabilité de grève.

b) Avantages pour l'employé

1) elle lui donne l'assurance d'un salaire raisonnable quel que soit le patron qui l'emploie;

2) elle lui pcrmet d'établir son budget familial ;

3) elle lui assure un salaire, qui sagement employé lui permet de vivre convenablement dans le présent et d'amasser pour l'avenir ;

4) elle rend plus amicalcs ses relations avec les patrons étant donné que tout sujet de désaccord aura été auparavant écarté ;

5) elle groupe plus solidement les gens d'un même métier, afin d'empêcher que des incompétents, alléchés par le salaire, n'envahissent la profession ;

6) amenant plus d'argent au foyer, elle contribuera pour une petite part à mettre de l'union dans la famille de l'ouvrier, parce que la misère, surtout la misère qui demeure, n'est pas bonne conseillère ;

19. Girard, Léonce, in La Vie Syndicale, vol. XX, n² 2, octobre 1935, p. 1. 
7) elle fait disparaître de l'esprit de l'ouvrier le cauchemar de la grève;

8) elle enlève à l'employé soucicux de ne pas mourir de faim l'idée de se faire complice de son patron pour accepter un salaire inférieur à celui établi, et par là nuire à ses confrères de travail ${ }^{20}$.

Il est intéressant de noter jusqu'à quel point les avantages définis par cette loi pour l'employeur diffèrent dans leur sens de ceux définis pour les employés. Quant à l'employeur (a), on lui démontre que cette loi dans son application ne peut que lui rapporter un meilleur rendement et aussi une meilleure utilisation des facteurs de production ct notamment de la force de travail (art. 2-3). Elle permet en même temps de diminuer la concurrence, surtout celle des salaires (art. 1 et 4). D'où les avantages que la loi prévoit pour les patrons sont d'ordre économique. Quanl: aux employés (b), les avantages prévus par la loi et définis par le syndicalisme catholique relèvent $\mathrm{du}$ psycho-sociologisme. On parle de salaire raisonnable sans définir en quoi il consiste (art. 1-2-3-8), de relations amicales entre patrons et ouvriers (art. 4), de solidarité de métier (art. 5), d'union dans la famille (art. 6) et de cauchemar de la grève (art. 7). Tout ce langage que l'on peut qualifier de normatif, traduit pour le syndicalisme catholique les orientations de cette loi appliquée aux travailleurs.

\subsubsection{L'extension juridique de la convention collective et l'aplanissement des rapports entre le capital et le travail}

Les conventions collectives à cxtension juridique visent à éliminer tous les motifs de tension entre le capital et le travail. Elles visent à supprimer dans leur source même les sujets de désaccord.

Parce que l'ordre est supérieur à la liberté, nous estimons qu'on doit organiser la convention collective obligatoire $"$.

Cette argumentation situe l'organisation des travailleurs au niveau des rapports sociaux avec l'employeur et ne fait jamais appel dans l'explication des orientations qui guident ces rapports patrons-employés à la structure et à l'organisation économiques de la société capitaliste, en tant qu'infrastructure de l'organisation sociale. Le capitalisme comme tel n'est jamais remis en question, il est le point de départ à partir duquel les rapports sociaux sont aménagés. Remettre en question la structure économique c'est permettre l'implantation du communisme et du socialisme ce que veut combattre le syndicalisme catholique :

l'élite ouvrière syndiquée ne doit pas s'opposer à cette loi. Ello doit poser un geste de charité chrétienne, de haute charité sociale. Cette loi consolidera le syndicalisme professionnel tant ouvrier que patronal. Cette généralisation des contrats arrêtera l'infiltration communiste $\stackrel{2}{2}$.

\subsubsection{L'organisation professionnelle et la lutte contre le communisme}

L'organisation professionnelle est considérée par le syndicalisme catholique comme la base fondamentale de regroupement des travailleurs et permet de mieux combattre les principes socialistes et communistes :

20. Lacombe, Léandre, aumônier des Syndicats de la Construction, in La Vie Syndicale, vol. XIII, $\mathrm{n}^{\circ} 9$, novembre $1933, \mathrm{p} .1$.

21. Boileau, Aimé (l'abbé), in La Vie Syndicale, vol. XIII, n 11, janvier 1934, p. 1.

22. Charpentier, Alfred, in La Vie Syndicalc, vol. XIII, $\mathrm{n}^{\circ} 12$, février 1934, p. 1. 
Les syndicats des travailleurs de la chaussure savaient depuis longtemps que la loi de l'extension juridique de la convention collective était entièrement opposée à tous les principes socialistes et communistes. Au lieu en effet de remettre tout dans les mains de l'État comme le veulent les communistes, elle laisse aux organisations professionnelles le soin de régler toute question de travail. Il est donc facile à comprendre que la Fédération industrielle de la chaussure, affiliéc à la Workers Unity League et soutenuc par elle, heurte de front les principes communistes.

La coopération entre patrons et ouvriers et la généralisation du bien-être dans la classe ouvrière, sont, en cffet, dcux obstacles à limplantation du socialisme et du communisme $=$

Il est fait allusion ici aux syndicats de la chaussure attachés aux unions internationales, d'orientation communistc.

La loi de la convention collective est en fait un instrument d'ordre dans la profession et de plus grande justice pour les employés et les employeurs. C'est un moyen de combattre l'anarchic professionnelle ${ }^{2 t}$.

La législation ouvrière et l'organisation professionnelle sont considérées par la doctrine sociale de l'Église comme instruments nécessaires à l'instauration de l'ordre social. Le syndicalisme catholique québécois reprend le principe en ces termes :

Depuis deux ans surtout, c'est-ì-dire depuis que la législation ouvrière donne un meilleur appui à l'action de l'Église et à l'action des corps professionnels, la C.T.C.C. a connu une expansion qui a continuellement tenu en haleine les chefs du mouvement dans les différents centres. Cette constatation met bien en évidence la pensée des encycliques Rerum Novarum et Quadragesimo Anno sur la simultanéité d'application nécessaire des trois remèdes proposés pour restaurer l'ordre social : religion, législation et organisation professionnelle ${ }^{25}$.

\subsubsection{La loi de l'extension juridique comme réponse à la situation de crise économique}

... Il resterait un vrai remède à appliquer pour assurer plus de prospérité permanente à la classe ouvrière ct éviter une lamentable déchéance des salaires en temps de crise économique, ce scrait l'extension juridique de la convention collective de travail ${ }^{20}$.

Parmi les autres remèdes mentionnés par Charpenticr se retrouvent $1^{\circ}$. «la présence du prêtre »qui «est un précieux encouragement et une source de bons conseils» et $2^{\circ}$. "les cartes de chômage» qui permettent aux chômeurs de demeurer membres du syndicat $: 2$.

23. Charpentier, Alfred, in La Vie Syndicale, vol. XIX, n ${ }^{\circ} 13,12$ mars 1935, p. 12.

24. Picard, Gérard, sec.-gén., in Rapport dı Bureau Confédéral, Congrès C.T.C.C., avril 1935, Procès-verbal, p. 59.

25. Poulin, Thomas, in La Vie Syndicale, vol. XIII, n 11, janvier 1934, p. 4.

26. Charpentier, A., Programme Souvenir, Fête du Travail de la C.T.C.C., septembre 1931, p. 5.

27. Idem. 


\section{CONCLUSION}

La loi de l'extension juridique de la convention collective est l'œuvre principalement du syndicalisme catholique qui, par ce biais, concrétise dans une réglementation législative des rapports patrons-ouvriers, les orientations qui sont celles de l'idéologie corporatiste. Les idéologues identifiés de la C.T.C.C. sont ceux-là mêmes qui interviennent directement dans la formulation de la loi et auprès du gouvernement pour la faire accepter. La loi de l'extension juridique assure une des bases essentielles de «l'ordre corporatif » et se veut un instrument de combat contre l'individualisme et le libéralisme économique. La négociation collective qui est le nœud de cette loi règle les rapports patrons-ouvriers dans le cadre de la collaboration, de la bonne entente et non de l'opposition et de la lutte des classes. D'où la loi intervient comme mécanisme de régulation assurant la pénétration de l'idéologie dans l'exercice des rapports sociaux fondamentaux propres à la société capitaliste : les rapports patrons-ouvriers. Lorsque les promoteurs de la loi font appel à la négociation collective comme moyen pour les patrons et les ouvriers «d'organiser mutuellement l'économie de leur industrie » et à l'extension juridique comme moyen d'assurcr l'organisation de l'économie au niveau d'une région donnée ou d'une industrie, ils orientent par le fait même le syndicalisme catholique vers un syndicalisme d'intégration.

Il s'agit d'un syndicalisme engagé dans une action de développement économique et de contrôle des conditions de travail et d'emploi... L'expression du mécontentement, l'organisation de la défense collective et l'action politique tendent alors à se confondre, puisque le syndicat cherche moins à mobiliser le nuécontentement qu'à participer au bon fonctionnement de la société. Le mouvement ouvricr peut ainsi devenir un élément d'un système totalitaire ou, plus modérément, une organisation animée par un modèle rationalisateur, mais qui peut fonctionner au niveau le plus bas aussi bien qu'au niveau le plus élevé... :

Pour êtrc plus juste, il nous faut préciser que les caractéristiques qui spécifient le syndicalisme catholique des années ' 30 , tant au niveau de son idéologie qu'au niveau de sa pratique syndicale le rapprochent davantage du type «syndicalisme d'intégration » que des autres types de syndicalisme identifiés par Touraine.

Il ne possède pas cependant tous les traits caractéristiques de ce syndicat d'intégration mais peut-être n'avons-nous pas su les identifier correctement à cause des lacunes dans notre cueillette de données. Ainsi « l'absorption de l'insatisfaction ouvrière par la revendication » dont parle Touraine ne peut faire l'objet de notre recherche vu l'impossibilité de procéder à une enquête ouvrière. Cependant, plusieurs indices nous conduisent à cette conclusion qu'il s'agit d'un syndicalisme d'intégration comportant quelques éléments propres au syndicalisme de négociation (dont le niveau des revendications ouvrières). Les indices peuvent s'identifier ainsi :

1. encadrement du syndicalisme catholique par la petite-bourgcoisie au niveau de son organisation centrale.

2. récupération par la classe ouvrière du discours des idéologies dominantes dans la société québécoise : le corporatisme et lc nationalisme - Récupération assurée par l'intermédiaire de la petite-bourgeoisie.

28. Touraine, Alain, Sociologie de l'acrion, Ed. du Seuil, 1965, p. 366. 
3. intégration du «projet syndical qu'est la C.T.C.C. » dans le Programme de Restauration sociale qui touche le contrôle du développement de l'ensemble de la société et Jes modes de participation à ce développement.

4. le rôle central accordé à l'État en tant que juge et arbitre des partics mises en cause par le développement du capitalisme.

Quant à l'aspect «syndicalisme de négociation \#, de la C.T.C.C., il apparaît surtout dans les résolutions votées en Congrès ct dans lc modc d'intervention auprès du patronat pour la réglementation des conditions de travail. Il recouvre davantage un mode d'intégration à la société capitaliste qu'un mode de contestation de celle-ci.

\section{LE CONGRÈS DES MÉTIERS ET DU TRAVAIL DU CANADA (C.M.T.C.) ET L'EXTENSION JURIDIQUE DE LA CONVENTION COLLECTIVE}

Autant les orientations normatives qui guident laction du syndicalisme catholique, nommément à propos de l'adoption et de la mise en application du système de l'extension juridique de la convention collective, nous apparaissent intégrées les unes aux autres et en parfaite continuité avec l'idéologic corporatiste qui constitue leur base d'unification, autant lorsque nous abordons l'analyse des orientations de la C.M.T.C., elles ne nous apparaissent pas soumises à première vue à la même cohérence, à la même unité. Cette division se retrouve non seulement au sein des orientations comme telles mais au niveau des leaders syndicaux et des différentes fédérations syndicales. Nous allons tenter dans l'analyse qui suit de vérifier s'il y a vraiment opposition à l'intérieur du système des orientations qui guident l'action du C.M.T.C. ou s'il s'agit davantage d'une image qui se dégage à première vue et qui ne persiste pas au niveau de l'analyse. Dans le mouvement marqué par une volonté de transformer l'organisation des travailleurs d'une part et l'organisation des rapports entre les ouvriers et leurs patrons d'autre part, la place et le sens dans lequel s'inscrit le syndicalisme québécois affilié aux unions internationales ne s'assimilent pas au rôle et aux orientations dans l'action du syndicalisme catholique québécois. Alors que ce dernier se fait l'instrument de défense de ce nouveau type de négociation collective auprès du gouvernement ct de la société québécoise, le C.M.T.C. pour sa part se voit devant un projet déjà constitué auquel il n'a pas pris part dans la phase d'élaboration, à qui on demande d'entériner le principe et de voir à son application. L'on verra comment il était impensable qu'une telle chose se produise sans susciter des oppositions profondes, lorsque l'on sait jusqu'à quel point et comment dans plusieurs de ces aspects, l'idéologie du syndicalisme catholique diffère de celle du syndicalisme international nord-américain.

Pour faire l'analyse du C.M.T.C. et de sa position, nous reférons à son journal syndical officiel, "Le Monde Ouvrier» et aux Procès-Verbaux de congrès et d'assemblées, sculs documents encore accessibles de nos jours. 
IDÉOLOGIE ET PRATIQUES SYNDICAIES AU QUÉBEC.

\subsection{LES IDÉOLOGUES DU C.M.T.C.}

\subsubsection{Socius ou Gustave Francq}

Les orientations qui guident certains leaders syndicaux du C.M.T.C. et définissent le sens de leur pratique (en regard du système d'extension des conventions collectives) peuvent se résumer assez grossièrement de la façon suivante (considérons ce raisonnement comme un point de départ de l'analyse) :

En temps normal, les unions ouvrières de par leur force économique maintienrent un certain équilibre;

en temps de crise, ccla leur devient difficile sinon impossible, les contrats de travail qu'elles passent avec leurs employeurs no couvrent pas un champ d'action assez vaste pour influer sur le marché du travail mais par contre mettent l'employeur qui les accepte dans une position d'infériorité vis-à-vis cles autres patrons qui ne sont liés par aucun engagement et qui paient à leurs

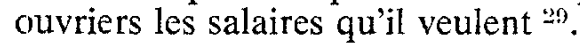

D'où l'extension de la convention collective présentée par le ministre Arcand, non pas comme une panacéc universelle, mais comme le meilleur moyen de relever les salaires et de mettre fin à une concurrence effrénée «apparaît comme tout à fait acceptablè à un certain nombre des membres des unions internationales. Cette position exprimée et défendue par Socius (pseudonyme) dans plusieurs articles du Monde Ouvrier reflète la tendance minoritaire voulant entériner le projet de loi du Ministre du Travail, projet issu des propositions de la C.T.C.C. Etant donné la situation de crise dans laquelle se trouve le Québec, cette tendance considère que le «Travail Organisé » ne peut pas « de par sa force économique seule dicter au patronat des contrats de travail $\gg$ et qu'avant de se prononcer sur le projet du Ministre du Travail et des Syndicats Catholiques il est conseillé aux syndicats « de ne pas condamner le projet à priori, de l'étudier à fond, d'y soumettre des amendements si nécessaire, car la législation dépendra de ce qu'on la fera:

Pouvons-nous descendre plus bas que nous le sommes actuellement? Les salaires peuvent-ils être plus avilis qu'ils ne le sont? Si nous n'avons plus rien à perdre, que peut-il nous arriver de pire ?

Si le Travail Organisé refuse au Ministre du Travail sa coopération, il devra en porter toute la responsabilité ; s'il considère que par sa seule force économique il peut dicter au patronat des contrats de travail, l'extension juridique des dits contrats ne l'affectera pas; mais s'il reconnaît qu'il ne le peut pas, alors le projet de la loi Arcand devrait rencontrer son approbation et sa coopération la plus active, c'est du moins notre manière de voir ${ }^{31}$.

L'orientation qui guide la prise de position de la tendance minoritaire peut être considérée comme relevant de la catégorie "réponse à une situation donnéc». Alors que dans les orientations qui ont guidé l'action du syndicalisme catholique nous avons trouvé un double mouvement : le système d'extension de la convention collective étant pour la C.T.C.C. une forme d'organisation des travailleurs ct des rapports patrons-ouvriers qui est à la fois une réponse à une situation donnée et l'expression d'une volonté de contrôle du développement de la société québécoisc, nous retrouvons en ce qui concerne les orientations de la tendance minoritaire du C.M.T.C., celles qui s'apparentent à "la réponse à une situation donnée ». L'autre

29. Socius, in Le Monde Ouvrier, vol, $20, \mathrm{n}^{\circ} 6,10$ février 1934, p. 1 .

30. Socius, in Le Monde Ouvrier, vol. $20, n^{\circ} 6,10$ février 1934, p. 1.

31. Socius, in Le Monde Otwrier, vol. 20, $\pi^{\circ} 6,10$ février 1934, p. 1. 
type d'orientations qui rejoint celle de la tendance majoritaire exprime un refus d'endosser ce projet d'extension juridique des conventions collectives et s'y oppose avec vigueur.

\subsubsection{Le Journal : Le Monde Ouvrier}

Il semble que les opposants à la loi de l'extension juridique des conventions collectives trouvent leur principal argument dans ce que représente pour eux l'obligation d'incorporation des différents syndicats qui veulent négocier des conventions collectives en vertu du système de l'extension juridique. Pour ceux qui s'inscrivent contre cette orientation d'incorporation, le syndicat ou l'ensemble des syndicats représentent une force économique qui doit s'imposer comme telle au niveau des rapports patrons-ouvriers. Pour eux il n'est pas question d'appuyer ou de se prononcer en faveur de cette loi dont l'incorporation marque le fondement.

Pour ceux qui appuient la passation de la loi de 1934, il apparaît qu'en situation de crise, le syndicat ne puisse se définir de par sa seule force économique et qu'il doive, par le fait même, recourir à un intermédiaire entre lui et le patron pour accroitre son pouvoir intermédiaire. C'est alors qu'une législation du travail contrôlée par l'État trouve sa légitimité.

Dans les circonstances, lorsque les unions ouvrières sont incapables de passer des contrats collectifs de travail avec les patrons, cette extension-reconnaissance légale d'ententes intervenues entre les deux parties - est certes une bonne chose, elle protège les salaires de l'ouvrier aussi bien que les intérêts des patrons. Elle met frein à la concurrence injuste ct déloyale par le fait qu'elle uniformise les salaires dans l'industrie en cause... Ce fut toujours le rôle des unions ouvrières de maintenir l'équilibre des salaires autant que faire se pouvait, soit en diminuant les heures de travail, soit par sa force économique. Lorsque cette force économique ne peut plus s'exercer pour le bien de l'industrie, le salaire s'avilit et plus il s'avilit, plus la dépression s'accentue. C'était bien là la situation dans la plupart des corps de métier sinon dans tous. Cette législation de l'extension remplace en quelque sorte cette force économique que nous avons perdue, elle permet aux patrons et aux organisations ouvrieres de se rencontrer, de chercher à s'entendre et de fixer des minima de salaires $q u i$, une fois légalisés, deviennent obligatoires même pour ceux qui n'ont pas été partie contractante à cette entente dans tous les districts où sa juridiction s'étend ... ${ }^{32}$

... aucune puissance du monde ne peut empêcher un citoyen qui se rend compte de l'exploitation de dire qu'un système social qui permet de telles iniquités est pourri jusqu'à la moclle et que le plus vite il disparaîtra le mieux ce sera pour le genre humain. Comment faire échec à ce dévergondage d'exploitation, de vol et de rapine ? On ne pourra y arriver que par un code de législation bien défini qui supprimera les gros profits, réglementera les prix de vente et fera régner une justice sociale basée sur les grands principes du christianisme et de l'honnêteté: $3:$.

Pour la tendance minoritaire, l'État apparaît donc comme l'instrument essentiel qui a comme rôle reconnu d'administrer les rapports patrons-ouvriers. Ce rôle ne lui est cependant pas reconnu de façon permanente mais lui revient en situation de crise économique; c'est l'État qui, alors, se doit d'exercer son rôle supplétif.

32. Socius, in Le Monde Ouvier, vol. 20, n²3, juin 1934, p. 4.

33. Socius, in Le Monde Ouvrier, 30 juin 1934, p. 1. 
Une des formes que peut prendre l'exercice de son rôle supplétif est l'intervention du pouvoir législatif :

L'équilibre entre le pouvoir d'achat et le coût de la vie a été rompu, il faut le rétablir et ceci ne peut se faire à moins que l'Etat n'intervienne et essaie tout au moins de remédier à cette situation déplorable, c'est ce qui a motivé l'adoption d'une loi de salaire minimum pour les femmes, et celle relative à l'extension des conventions collectives de travail; ces deux lois, tendent à ctablir des rapports plus cordiaux entre patrons et employés en les rapprochant l'un de l'autre en des conférences paritaires ou conjointes ... Ces innovations dans notre législation sociale sont un pas en avant vers la transformation du régime capitaliste actuel en un système plus humanitaire et plus rationnel où les dividendes du travail primeront ceux du capital pour le plus grand bien de l'humanité entière ${ }^{3 t}$.

Ce qui nous fait penser que la position de la tendance minoritaire concernant le rôle de l'État en est une de situation, c'est que lorsque aux États-Unis, la loi Wagner, adoptéc à la session du Congrès en 1934, fut, l'année suivante, déclarée inconstitutionnelle (loi qui reconnaissait, rappelons-le brièvement, le droit aux travailleurs de s'organiser légalement sans avoir à subir la contrainte ou l'intervention de leurs patrons), le rédacteur-en-chef du Monde Ouvrier, porte-parole de cette tendance, face à ce problème, s'exprima ainsi :

Il ne fallait pas s'attendre à ce que le patronat américain, et sur ce point, il est le même partout, se laisse dépouiller de son droit d'exploiter la classe ouvrière, lui qui jouit de toutes les libertés qu'il refuse obstinément de reconnaître aux autres...

Cet incident nous rappelle que le dicton qui dit qu'on n'est jamais si bien servi que par soi-même est toujours vrai. Les ouvriers ne parviendront à obtenir la reconnaissance de leurs droits qu'en s'organisant et ils ne doivent pas compter sur les textes de loi pour se faire une place au soleil. Quand ils sauront s'en servir, ils n'auront plus besoin de recourir aux gouvernements pour leur bien-être ${ }^{35}$.

Cette attitude face à la situation américaine nous apparaît être un indice important qui nous permet de poser l'hypothèse à savoir que la position de la tendance minoritaire face au système de l'extension juridique de la convention collective n'est pas irréconciliable voire contradictoire avec celle de la tendance majoritaire qui elle s'oppose à l'adoption de ce système. Socius et ceux qui pensent comme lui voient dans cette loi une réponse et presque la seule à la situation de crise dans laquelle se trouvent les sociétés capitalistes en 1929. Ils considèrent cette solution comme un tampon, la situation socio-économique ne pouvant se dégrader davantage. Les conséquences de la crise sont telles que tout moyen quel qu'il soit ne peut sans doute pas aggraver les conditions de travail des ouvriers mais pourrait peut-être en revanche améliorer leur position économique. Ils admettent donc l'intervention de l'État à certains moments du développement d'une société, et la crise économique constitue l'un de ces moments. Ils ont proposé plusieurs amendements, le contenu de la loi leur apparaissant nettement insuffisant.

34. Francq, Gustave, président de la commission du Salaire minimum des femmes du Québec, conférence à CKAC, 18 janvier 1935, rapportée dans Le Monde Ouvrier, 19 janvier 1935 , p. 1.

35. Socius, in Le Monde Otvrier, 28 décembre 1935, p. 1. 
Ils appuient la C.T.C.C. dans sa demande au gouvernement provincial, « de cesser la propagande en faveur de la loi des salaires raisonnables et de favoriser l'application de conventions collectives de travail ».

Nous en sommes, mais à la condition toutefois que ce soient de véritables conventions collectives qui couvrent l'industrie entière et interviennent entre une association patronale et une organisation ouvrière bona fide composée de membres payant leurs contributions et en règle avec elle.

Nous sommes également d'accord pour condamner les tentatives faites par un représentant du Ministère du Travail pour former les comités d'usines et empêcher de ce fait lenrôlement des ouvriers dans des organisations ouvrières ${ }^{36}$.

Le critère «juridiction territoriale » est mis en cause par les Unions Internationales qui lui opposent le critère «secteur industriel» comme base valide d'une véritable convention collective.

\subsubsection{Opposition ville-campagne - opposition grands centres industriels - petits centres}

Nous estimons que ceci est un danger pour les grands centres où les unions ouvrières sont, sinon florissantes, tout au moins en existence tandis qu'elles sont l'exception dans les petits centres industriels et les districts ruraux; il en résulte que si un contrat collectif exige le salaire d'union à Montréal, celui-ci sera de beaucoup plus élevé que celui des autres endroits et l'industrie ira se réfugier où il n'y a pas d'organisation ouvrière ou bien il arrivera que des patrons avisés organiseront en sous-mains un embryon d'association ouvrière qui négociera un contrat collectif à taux réduit...

Il semble que comme mesure de protection pour les grands centres, les contrats collectifs intervenus dans les petits centres devraient être sur la base de ceux de Montréal avec un certain pourcentage fixe en moins pour les autres villes de la province, d'après leur chiffrc de population, ou dans un certain périmètre $\bar{~} \overline{\text {. }}$.

Ceci est un point capital ct mérite qu'on le relève afin de bien montrer comment la loi do 1934 n'est finalement qu'une mesure réformiste de peu de portée quant au problème auquel clle s'attaque. Si on l'étudie au niveau de sa pratique (nous le ferons ultérieurement), on se rend bien compte qu'étant donné le caractère facultatif du recours au système d'extension juridique des conventions collectives, cette dernière mesure possède un impact affaibli au point de départ et réduit de beaucoup ses possibilités de répondre aux problèmes pour lesquels elle a été créée, soit la crise économique ct ses conséquences: la compétition économique, la concurrence industrielle, le chômage accru... Nous verrons que la catégorie de ceux qui recourent à cette mesure comprend beaucoup plus les petits industriels canadiens-français, affiliés à la C.T.C.C. et qui se regroupent dans les petites et moyennes entreprises que les gros industriels, les grands capitalistes qui possèdent la majorité des grandes entreprises. Ces derniers se sont opposés à cette mesure ct n'en ont à peu près pas tenu compte, alors que c'est à ce niveau que des mesures comme la loi de 1934 auraient dû s'appliquer, à cause des répercus-

36. Le Monde Onrier, 9 octobre 1937, p. 1.

37. Socius, in Le Monde Ourrier, 6 avril 1935, n. 1. 
sions de la concurrence sur les petites et moyennes entreprises canadiennesfrançaises vivement secouées par la crise. Nous nous retrouvons devant le prénomène inverse : ce sont les petites et les moyennes entreprises qui recourent à la loi de '34 pour mieux se protéger, en se regroupant, contre l'envahissement au niveau du développement économique, de la grande entreprise :

... la lutte économique d'aujourd'hui a changé de terrain, ce n'est plus tant 1c patron qui lutte contre l'ouvrier et vice-versa, mais c'est le manufacturier des grands centres qui doit lutter contre celui des districts ruraux. C'est un des beaux résultats de la décentralisation de la grande industrie ${ }^{38}$.

\subsubsection{Opposition « démocratie industrielle»-corporatisme}

Le corporatisme, pour les unions internationales, représente un système social inacceptable au niveau de l'organisation des rapports patrons-ouvriers. C'est aller contre ce qu'elles appellent «la démocratie industrielle», système social auquel font beaucoup référence, surtout après 1935, les syndicats américains.

La négociation collective considérée en tant que pratique spécifique de la démocratie industrielle, marque le passage de la relation individuelle entre l'employeur et l'employé, «symbole de l'ancien ordre social» à un «nouvel ordre social » marqué par l'exercice d'une «politique de démocratie dans l'industrie, de la liberté économique et de la négociation collective ${ }^{39} \gg$.

La négociation collective n'a pas pour but de créer un conflit de classe ou une conscience de classe. Ses buts sont à linverse et tendent à amener employeurs et employés ensemble pour qu'ils discutent leurs intérêts conflictuels et agissent suivant leur intérêt mutuel... Le droit à la grève demeure un droit fondamental tout comme celui de posséder, contrôler et diriger la propriété dans un pays capitaliste ${ }^{\text {th }}$.

Dans une réponse de protestation adressée au ministre du travail, W. Tremblay qui déclarait que le gouvernement québécois, dirigé par le premier ministre Duplessis, ne tolérerait plus les ateliers fermés, les unions internationales par la voix de leur Journal, Le Monde Ouvrier, ont exprimé la position suivante dans laquelle elles rappellent leur opposition au corporatisme et leur appui à un système dit de «démocratic industrielle»:

Jusqu'ici on peut le dire sans crainte, l'exploitation des ouvriers était évitée seulement lorsque l'union pouvait établir un système de contrôle efficace. Les unions internationales depuis plus de $3 / 4$ de siècle mettent en pratique le contrat collectif et l'atelier fermé. Ce principe a toujours fait partie de la déclaration de principes des unions internationales et des syndicats catholiques.

Les ouvriers voient avec méfiance les nouvelles lois sociales (ouvrières) qui viennent d'être adoptées et voient distinctement qu'elles les mènent au corporatisme, au facisme ct tỉnalement à la dictature propre à Mussolini, c'est-à-dire la dictature industrielle, à laquelle ils s'opposeront de toutes leurs forces. Les travailleurs bataillent pour leur liberté économique au moyen de droits légaux d'appartenir à l'union de leur choix, de passer des contrats collectifs et d'obtenir l'atelier fermé, de faire la grève contre l'exploitation et ils ne toléreront pas que les autorités provinciales leur mettent des cntraves pour

38. Socius, in Le Monde Ourrier, 4 août 1934, p. 1.

39. Green, W., Organizational Freedom in Le Monde Ouırier, 6 août 1938, p. 7.

40. Idem. 
nier les droits qu'il possèdent dans le pays depuis un temps immémorial et qui, s'ils leur sont refusés, auront tendance à faire disparaître les unions ouvrières ${ }^{41}$.

Le système corporatiste tient lieu, pour les unions internationales de « dictature économique », d'ingérence outrée de la part du gouvernement, d'un gouvernement qui défend certains objectifs politiques qui sont en contradiction avec la défense des droits des travailleurs.

Pour les unions internationales, l'incorporation c'est l'intervention du gouvernement dans la réglementation des rapports patrons-ouvriers; c'est aussi le syndicat qui s'en remet au gouvernement pour la défense de ses droits alors que les deux s'opposent l'un à l'autre ; c'est l'abandon par le syndicat de son rôle véritable qui en est un de lutte économique à partir d'une action menée par les seuls travailleurs s'appuyant sur leur organisation. Au lieu de donner plein pouvoir au Ministre du Travail et à son gouvernement et en dernier lieu, au Lieutenantgouverneur, il aurait mieux valu, pour éviter les abus qui s'ensuivent, "laisser à un organisme absolument indépendant, soit un Conseil Supérieur du Travail ou une commission paritaire, genre conseil de Prud'homme - le soin de décider en dernier ressort des conventions collectives et des ordonnances de l'Office des salaires raisonnables, si réellement un contrôle est nécessaire, ce que nous ne sommes pas prêts à admettre ${ }^{42} \gg$.

Les syndicats internationaux se méfient du régime Duplessis. Pour ceux-ci, le gouvernement Duplessis en est un de « quasi-dictatorial en matière de législation sociale, il possède une attitude anti-ouvrière ${ }^{43} \gg$. Souvent il est fait appel à un front commun ouvrier des syndicats catholiques et des unions internationales pour combattre le régime Duplessis. Le premier Ministre Duplessis a été violemment attaqué par les organisations syndicales à cause de sa politique anti-ouvrière à l'égard des chefs syndicaux.

Les catégories de syndiqués qui, à l'intérieur des unions internationales appuient la loi de l'extension juridique de la convention collective se regroupent à l'intérieur de la même phase du développement de l'organisation du travail, soit (selon la théorie de Touraine) la phase $A$, phase où le travail est essentiellement fondé sur le métier. Il est intéressant de rappeler par ailleurs que le syndicalisme catholique pour sa part recrute à cette époque essentiellement parmi les ouvriers de métiers et très peu parmi les ouvriers industriels. Cette observation nous oblige à complexifier notre hypothèse de départ, à savoir que l'idéologie qui guide l'action syndicale de chacune des organisations de travailleurs syndiqués n'est pas le seul facteur d'explication de la position de celle-ci face au système de l'extension juridique des conventions collectives. Car comment expliquer alors qu'il y ait divergence à l'intérieur des unions internationales face à cette loi qui propose un nouveau système de réglementation des rapports patrons-ouvriers? Il faut pour le comprendre recourir à une autre variable d'explication. Les années 1930-40 au Québec (et surtout après que les plus gros effets de la crise économique se font

41. Hachette, R., in Le Monde Ouvrier, 5 juin 1937, p. 4.

42. In Le Monde Otvrier, 29 mai 1937, p. 1.

43. Francq, Gustave, Demain in Le Monde Ouvrier, 3 mars 1938, p. 8. 
moins sentir) sont marquées par un deuxième mouvement d'industrialisation, caractérisé par le passage définitif de la phase $\mathrm{A}$ (fondée sur le métier) à la phase $\mathrm{B}$ (fondèe sur l'industrie). C'est l'industrie regroupant des travailleurs spécialisés, des travailleurs effectuant des tâches parcellaires qui remplacent peu à peu les ouvriers de métier. Or, si l'on examine de plus près les catégories de travailleurs qui se rallient autour de la loi de '34, et qui votent en faveur de son adoption, nous remarquons que ce sont 1 . ceux, des syndicats catholiques, considérés globalement et d'où cette loi émane en tant que pratique du corporatisme, 2 . ce sont en même temps ceux des moyennes et des petites entreprises où recrute surtout le syndicalisme catholique à qui se joignent les patrons qui sont en majorité canadiens-français et qui voicnt dans ce système de négociation un moyen de se défendre contre la concurrence des grandes entreprises en majorité étrangère, 3. ce sont, pour une majorité, les ouvriers de métiers, rattachés en tant que syndiqués au syndicalisme catholique ou aux unions internationales, qui se sentent visés par la croissance rapide de l'industrialisation, qui voient leur métier menacé et appelé à disparaître ; ils se raccrochent à la loi de 1934 , comme moyen de se protéger contre les dangers de disparition et de concurrence que présentent pour eux les travailleurs industriels et les exigences de spécialisation des grandes industries :

Dans les années 1930-1940, nous assistons à une transformation importante de la structure économique et parallèlement à une transformation de la pensée et de l'action syndicale. Le syndicalisme industriel ou «horizontal », constitué de tous les ouvriers préposés à une industrie donnée, indépendamment des métiers qui y sont exercés, remplace le syndicalisme professionnel ou «vertical». D'où la division qui se fait entre des syndicalistes qui sont rattachés à la formule traditionnelle et ceux qui s'orientent vers le syndicalisme industriel ${ }^{44}$.

\section{CONCLUSION}

Une fois de plus, lanalyse des positions et des interventions face à la loi de 1934 nous permet de dessiner plus précisément les contours idéologiques qu'épouse chaque organisation syndicale. Les positions que le C.M.T.C. adopte sont en continuité parfaite avec le cadre de référence global que nous avons identifié comme étant le libéralisme économique. La démocratie industrielle fonde de façon plus immédiate la pratique syndicale du C.M.T.C.. C'est ce qui explique que le C.M.T.C. soit, clans l'ensemble, réfractaire à la loi de 1934 ; elle ne permet plus aux syndicats d'intervenir dans le champ de développement du capitalisme sur la base de leur seule force économique et d'entrer dans le jeu «démocratique 》 du rapport de force. L'intervention de l'État comme tiers entre le patronat et le syndicat constitue l'obstacle majeur auquel se heurtent les unions internationales. Ells admettent cependant cette intervention étant donné la situation de crise économique qui prévaut à cette époque; dans ces moments de déséquilibre, le C.M.T.C. reconnaît à l'État le devoir d'exercer son pouvoir législatif et de suppléer au rapport de force qui ne peut, dans ces conditions, se jouer normalement. Tout en admettant ce rôle

44. Francq, G., L'évolution de la pensée syndicale, in Le Monde Onvier, 5 septembre 1936, p. 2. 
de l'État, le C.M.T.C. s'en prend cependant au caractère abusif de l'exercice de ce pouvoir permis par la loi de l'extension juridique. Le pouvoir absolu du lieutenant-gouverneur et la manipulation de la loi soumise à son bon vouloir soumettent les travailleurs aux intérêts patronaux défendus par les politiciens.

Le C.M.T.C. reconnaît dans l'État un instrument de défense des intérêts de la classe dominante (qu'il identifie comme étant composé majoritairement des patrons et des employeurs des entreprises) et il exige une refonte complète du Code Civil qui, dans son application, favorise « les intérêts patronaux au détriment des intérêts des ouvriers $»$.

Les positions du C.M.T.C. face à la loi de ' 34 sont à la fois investies par l'idéologie qui est sienne et par sa pratique syndicale. On se rappelle que la base de recrutement du C.M.T.C. est constituée surtout des ouvriers des grandes entreprises industrielles concentrées principalement dans la région de Montréal. Ces entreprises sont touchées directement par la loi qui prévoit une division territoriale sur la base de laquelle sont définies les conditions de travail et de salaire. Cette division territoriale consacre une opposition entre les centres urbains (principalement la ville de Montréal) et les centres ruraux, et entraîne, par les inégalités salariales entre les deux pôles, le jeu de la concurrence. Les industries de Montréal sont les plus touchées par cette loi, car elles doivent payer des salaires plus élevés, ce qui contribue à diminuer leur profit. La main-d'œuvre rurale se déplace vers la ville pour obtenir de meilleurs salaires et les propriétaires de capitaux et de moyens de production tendent à émigrer vers les campagnes où la force de travail s'achète à meilleur prix.

Selon le C.M.T.C., les inégalités salariales créent une division chez les travailleurs en entraînant une compétition entre les petites et les moyennes entreprises d'une part, et les grandes entreprises d'autre part. Cette concurrence ne peut être soutenue adéquatement par les petites et les moyennes entreprises qui se voient obligés de fermer leurs portes une à une pour céder la place aux grandes entreprises et aux monopoles. La main-d'œuvre suit le mouvement parallèlement, attirée par de meilleures conditions de travail. Les ouvricrs syndiqués du C.M.T.C. se recrutant en majorité, comme nous venons de le dire, dans les grandes industries montréalaises, ne reconnaissent pas dans la loi un moyen d'améliorer leurs conditions de travail spécifiques, et c'est pourquoi ils ne la soutiennent pas spécialement. Seuls les ouvriers de métier, menacés par le développement du capitalisme industriel qui les remplace par des ouvriers qualifiés et spécialisés, appuient cette loi avec ardeur. Elle leur assure des conditions de travail minimales qu'ils n'obtiendraient pas par le seul jeu de leur force économique. Cette loi qui se voulait un instrument de planification économique pour pallier aux effets de la crise en même temps qu'un moyen d'assurer des conditions de travail uniformes pour l'ensemble des travailleurs, et par là diminuer le taux d'exploitation de la force de travail, n'aura pas, à notre avis, au moment et pendant la durée de son application, répondu à ses objectifs. Nous le verrons dans l'étude des décrets et dans l'analyse des positions prises par les employeurs à son égard.

Nous pouvons tout de suite conclure, cependant, sur la base de l'analyse que nous venons de faire du C.M.T.C. ct de la C.T.C.C., que cette loi a surtout permis à des secteurs touchés directement par le passage du capitalisme concurrentiel au 
capitalisme monopoliste, ct menacés de disparaître à plus ou moins court terme, de s'assurer une survie momentanée en s'abritant sous l'aile de l'État. Ceci vaut autant pour les employeurs que pour les ouvriers de ces secteurs. C'est sur cette base uniquement que nous pouvons comprendre les appuis et les oppositions qui se sont maanifestés autour de cette loi tant au niveau de l'application qui en a été faite qu'au niveau idéologique.

En dernier lieu, disons que pour les unions internationales, la loi tue le principe même de la négociation collective fondée sur le libre jeu du rapport de force patrons-ouvriers dans unc entreprise donnée. L'extension à tout un secteur industricl et à toute une région économique d'une convention collective négociée dans une entreprise ainsi que l'intervention du lieutenant-gouverneur fausse «le jeu de la démocratie industrielle » dont l'instrument principal est la négociation collective entre deux parties.

Les unions internationales s'identifient davantage à un syndicalisme de négociation $^{45}$.

Le syndicalisme de négociation semble se réduire aux relations industrielles dans lesquelles il est engagé. Peut-être parce qu'il no met pas en cause un système de pouvoir économique qui lui semble d'une efficacité raisonnable, il ne prend pas en charge les problèmes du développement économique...

Dans cette situation, lc mouvement ouvrier tend à réduire les problèmes de l'action et ceux de la personnalité à leur signification stratégique dans un système de relations industrielles.

C'est moins en termes de développement que d'équilibre économique, de pouvoir de décision que de pouvoir de négociation, que sont saisies les orientations fondamentales du mouvement ouvrier. De même l'insatisfaction ouvrière n'est plus saisie qu'à travers l'examen des plaintes (procédure de grief) qui cherche à atteindre un certain équilibre des charges et des satisfactions plutôt qu'à transformer l'insatisfaction en mouvement social.

Les caractéristiques du syndicalisme de négociation identifiées par Alain Touraine ne se retrouvent pas toutes présentes au même degré dans le syndicalisme pratiqué par les Unions Internationales dans la période des années '30. Certaines sont dominantes, d'autres sont absentes. Ainsi, tout l'aspect de «l'activité politique indépendante ou autonome par rapport au mouvement ouvrier $»$ dont parle Touraine ne se présente pas de la même façon pour les Unions Internationales. Il faut cependant admettre que la période des années'30 est assez particulière à ce niveau, étant donné la présence plus ou moins organisée des communistes à l'intérieur du C.M.T.C. à cette période. La négociation des conditions de travail demeure cependant le lieu privilégié de l'action syndicale ct le moyen d'intervention par excellence sur la répartition des profits entre les patrons et les ouvriers d'une entreprise. L'action politique organisée n'est pas reconnue comme prioritaire par le C.M.T.C. en tant que syndicat et elle relève de la décision de chaque individusyndiqué. Le C.M.T.C., tout comme l'A.F.L., attaque le système capitaliste non pas dans ses racines mais dans ce qu'il appelle ses abus et il s'en remet à l'exercice de la clémocratie pour assurer l'équilibre des forces, conforme en cela à l'idéologie du libéralisme économique et de la démocratie industrielle.

45. Touraine, Alain, Sociologie de laction, p. 366. 


\section{LES DÉCRETS : MODE D'APPLICATION DE LA LOI DE L'EXTENSION JURIDIQUE DE LA CONVENTION COLLECTIVE}

Étant donné l'espace restreint qui nous cst réscrvé, nous ne pouvons présenter ici que les conclusions de l'étude que nous avons faite.

L'analyse de l'application de la loi de l'extension juridique de la convention collective, à travers le système des décrets, confirme l'hypothèse que nous avons émise préalablement à savoir que cette loi a surtout permis aux secteurs directcment touchés par le développement du capitalisme industricl à l'échelle de la grande entreprise et du monopole, de maintenir des conditions de travail et de salaires minimales acceptables pour les ouvricrs d'une part et, d'autre part, d'éviter, pour un temps seulement, qu'une trop grande concurrence accélère le processus de fermeture des petites et moyennes entreprises. De plus il apparait clairement que, sollicité par les syndicats catholiques d'une part, qui veulent absolument la passation de cette loi et, d'autre part, harangué par les Unions Internationales qui n'en veulent pas, l'État tranche le débat en assurant le recours facultatif à l'extension de la convention collective. Nous pouvons dire que l'intervention des patrons des grandes entreprises n'est pas étrangère à cette prise de position de l'État.

Il est vrai de dire que le pouvoir réside dans la personne du lieutenantgouverneur en conscil, en bonne partic, mais il ne faut pas oublier la fonction du comité paritaire dans l'application de la loi. Ce comité, composé d'un nombre égal de représentants patronaux et de représentants ouvriers, possède des pouvoirs formels reconnus légalement: pouvoir de rccours à la place des employés, contrôle sur les employeurs, attribution de cartes de compétences, surveillance de l'application du décret. Étant donné la nature de ces pouvoirs, il aurait été utile de procéder à l'étude empirique du fonctionnement des comités paritaires à travers la province pour saisir leurs formes d'intervention au niveau de l'application de la loi ; prenons un exemple: étant donné le pouvoir du comité paritaire d'exiger des cartes de compétence pour exercer tel ou tel métier, il est permis de poser l'hypothèse suivante, à savoir que ce pouvoir sc double d'un autre pouvoir qui est celui de régulateur de la circulation de la main-d'œuvre active sur le marché de l'emploi dans certains secteurs. Quels sont les critères qui servent de plate-forme à l'attribution des cartes de compétence? Est-ce qu'en période de chômage, il devient plus difficile pour les ouvriers d'obtenir ces cartes, étant donné que dans la pratique, elles constituent de véritables cartes de travail ?

Une autre question que nous aurions aimé aborder et à propos de laquelle il nous est impossible de recueillir des données, est celle du militantisme syndical. Nous pourrions la formuler ainsi : étant donné que le décret fixe d'autorité les conditions de travail à tout un ensemble de travailleurs qui n'ont pas négocié eux-mêmes de convention collective, est-ce que le degré de militantisme syndical demeure le même et cst-ce qu'il prend un sens différent, la négociation d'une convention collective n'étant plus son objectif principal ? Nous savons que cette loi a permis à la C.T.C.C. d'accroître considérablement ses effectifs, surtout dans les régions autres que celle de Montréal. A-t-elle permis un accroissement du nombre de syndiqués sans affecter le type de militantisme ou si la période qui a suivi immédiatement l'application de la loi a connu des fluctuations du militantisme chez les syndiqués (anciens et nouveaux)? 
Il nous serait utile de pouvoir répondre à cette question, étant donné que l'État a eu tendance à recourir au système des décrets après 1940, dans des périodes particulièrement marquées par un syndicalisme militant fort et par des grèves touchant des secteurs névralgiques du fonctionnement de la société québécoise ${ }^{46}$.

En ce sens, il nous semble que cette loi présentée sous le couvert d'une défense des intérêts des ouvriers sert davantage ceux de l'État et de la bourgeoisie industrielle dont les intérêts sont liés. La loi, considérée sous la forme des décrets, prend davantage l'aspect d'un instrument qui, dans les mains de l'État, permet le contrôle de la force économique des travailleurs organisés en syndicats.

Étant donné la situation particulière de la crise économique de 1929 , cette loi a, d'une part, permis à certaines catégories de travailleurs syndiqués d'obtenir des conditions de travail qu'ils n'auraient pu obtenir par la seule négociation collective; le système des décrets a, d'autre part, permis l'extension de ces gains aux travailleurs non syndiqués des secteurs couverts par l'application du décret.

Elle a permis à d'autres catégories de travailleurs et d'employeurs d'obtenir une réponse à leurs revendications liées dans leur ensemble à une situation socioécononique bien particulière. Étant donné son caractère facultatif et l'intervention prépondérante de l'État, cette loi ne peut être considérée comme un système d'organisation des rapports patrons-ouvriers axé prioritairement sur la défense des intérêts de tous les travailleurs. Elle constitue le résultat d'un compromis qui, à la fois, satisfait certaines revendications des travailleurs tout en protégeant les intérêts du patronat industriel et des employeurs du commerce et en laissant la voie complètement libre à la grande entreprise et aux monopoles étrangers dans leur insertion dans l'économie québécoise.

\section{RÉSUMÉ}

Nous avons choisi de faire l'analyse de la loi de l'extension juridique de la convention collective votée par le Parlement québécois en 1934. Cette loi nous intéresse en tant qu'indice de la pratique des rapports sociaux propre à une société capitaliste : les rapports entre l'État, la fraction industrielle de la classe bourgeoise et la classe ouvrière. Elle nous intéresse aussi par ce qu'elle permet de poser le rapport entre les idéologies et les pratiques syndicales du Québec des années * 30 ". Dans cette étude, nous avons pu rendre compte de cette loi en tant que transcription des idéologies dominantes d'une part mais aussi en tant qu'indice révélateur des orientations normatives et des intérêts politiques de la classe ouvrière, d'autre part. L'étude partielle des débats qui ont entouré cette loi, nous aura permis de mettre en place les forces sociales impliquées dans le développement de la sociêté québécoise de cette époque et de dégager le sens de leur implication.

\section{SUMMARY}

We have chosen to analyse the Act regulating collective bargaining which was passed by the Quebec parliament in 1934. This law is of interest as an indication of social relations in a capitalist society: the interaction between the state, the industrial segment of the bourgeoisie

46. La loi 89 que s'apprêtait à voter, en 1973, le Parlement dirigé par le parti libéral et qui enlevait le droit de grève à une majorité de travailleurs du secteur public et para-public tout en fixant par décret leurs conditions de travail, va dans ce sens. La négociation dans le secteur public qui se poursuivra à l'automne 1975 est à surveiller à la lumière de ces réflexions. 
and the working class. Another interesting aspect is the light it throws on the relationship between ideologies and union practices in the Quebec of the 30's. On one hand, we analysed the importance of this Act as a reflection of the main ideologies of the time; on the other hand we studied it as a significant indication of the normative trends and the political concerns in the working class. A partial analysis of the debates on the Act enabled us to situate the social forces at work behind the orientations of Quebec society at the time, and to analyse their significance.

\section{RESUMEN}

Hemos escogido hacer el análisis de la ley de la extensión jurídica de cl contrato colectivo (convention collective) adoptada por el parlamento de Quebec en 1934. Esta ley nos interesa en tanto que es un índice de la práctica de las relaciones sociales propias a una sociedad capitalista : las relaciones entre el estado, la fracción industrial de la clase burgesa y la clase obrera. Nos interesa también porque permite plantear la relacion entre las ideologías y las prácticas sindicales de Quebec de los años "30". En este estudio hemos podido dar cuenta de esta ley en tanto que transcripción de las ideologías dominantes por un lado pero también como índice revelador de las oricntaciones normativas y de los intereses políticos de la clase obrera, por otro lado. El estudio parcial de los debates que se han llevado a cabo en torno a esta ley, nos ha permitido, situar las fuerzas sociales implicadas en el desarrollo de la sociedad de Quebec de esta época, y destacar el sentido de su implicación. 